\title{
The relationship between maternal methadone dose at delivery and neonatal outcome: Methodological and design considerations
}

\author{
Hendrée E. Jones ${ }^{\mathrm{a}}$, Lauren M. Jansson ${ }^{\mathrm{b}}$, Kevin E. O'Grady ${ }^{\mathrm{c}}$, and Karol Kaltenbach ${ }^{\mathrm{d}}$ \\ aUNC Horizons and Department of Obstetrics and Gynecology, University of North Carolina at \\ Chapel Hill, Carrboro, NC 27510 USA; and The Departments of Psychiatry and Behavioral \\ Sciences and Obstetrics and Gynecology, School of Medicine, Johns Hopkins University, \\ Baltimore, MD 21224 USA, hendree_jones@med.unc.edu \\ bSchool of Medicine, Johns Hopkins University, Baltimore, MD 21224 USA, ljansson@jhmi.edu \\ 'Department of Psychology, University of Maryland, College Park, College Park, MD 20742 USA, \\ ogrady@umd.edu
}

dDepartments of Pediatrics and Psychiatry and Human Behavior, Jefferson Medical College, Thomas Jefferson University, Philadelphia, PA 19107 USA, karol.kaltenbach@jefferson.edu

\begin{abstract}
Compared to untreated opioid dependence, methadone maintenance treatment of opioid-dependent pregnant women has been found to be associated with better maternal and neonatal outcomes. Secondary analysis of data from 73 maternal and neonatal participants in the MOTHER study (H. E. Jones et al., New England Journal of Medicine, 2010) found no relationship between maternal methadone dose at delivery and any of 9 neonatal outcomes - peak neonatal abstinence syndrome (NAS) score, total amount of morphine needed to treat NAS, duration of neonatal hospital stay, duration of treatment for NAS, estimated gestational age at delivery, Apgar score at 5 minutes, and neonatal head circumference, length, and weight at birth. These results are consistent with a recent systematic review and meta-analysis (B. J. Cleary et al., Addiction, 2010) and extend findings to outcomes other than NAS. Methodological and design issues that might have adversely impacted the ability of researchers to establish the existence or non-existence of these relationships are considered.
\end{abstract}

(C) 2013 Elsevier Inc. All rights reserved.

Corresponding Author: Hendrée E. Jones, PhD, UNC Horizons, Department of Obstetrics and Gynecology, University of North Carolina at Chapel Hill, 400 Roberson Street, Carrboro, NC 27510 USA, Voice: 1-919-966-9803, Fax: 1-919-966-9169, hendree_jones@med.unc.edu.

Publisher's Disclaimer: This is a PDF file of an unedited manuscript that has been accepted for publication. As a service to our customers we are providing this early version of the manuscript. The manuscript will undergo copyediting, typesetting, and review of the resulting proof before it is published in its final citable form. Please note that during the production process errors may be discovered which could affect the content, and all legal disclaimers that apply to the journal pertain.

\section{Conflict of Interest}

HEJ has received reimbursement for her time and travel and KEO'G has received reimbursement for his time from Reckitt Benckiser Inc., manufacturer of buprenorphine. LMJ receives medication from Reckitt Benckiser Inc. for study-related purposes. KK reports no conflicts of interest related to this paper.

The authors alone are responsible for the content and writing of this article.

No honorarium, grant, or other form of payment was given to any author or any other individual to produce the manuscript.

All authors have approved the final manuscript. 


\section{Keywords}

opioid dependence; pregnancy; methadone; neonate; neonatal abstinence syndrome

\section{Introduction}

Methadone has been the most widely prescribed medication for the treatment of opioid dependence during pregnancy (Jones et al., 2006). Research over the past four decades has consistently supported its relative safety in the population of opioid-addicted pregnant women, and it has been adopted worldwide as the standard of care in this population (National Consensus Development Panel on Effective Medical Treatment of Opiate Addiction, 1998). Methadone pharmacotherapy during pregnancy reduces the risk of fetal exposure to the rapid and unpredictable cycles of highs and withdrawal and exposure to other toxins associated with heroin use. Moreover, methadone pharmacotherapy has been found to be superior to untreated heroin dependence on outcomes such as increasing the likelihood of obstetrical care, improved fetal growth and decreasing the risks of fetal mortality, HIV infection, and risk of preeclampsia [e.g., Finnegan (1991a,b); Jones et al. (2006); Kaltenbach et al. (1998); Kandall et al. (1977); Schmid et al. (2010)]. Methadone pharmacotherapy has also been found to be associated with superior treatment retention (Finnegan et al., 1992; Jones et al., 2001; Jones et al., 2008; Laken et al., 1996; Svikis et al., 1997) and less risk of relapse (Connaughton et al., 1977; Finnegan, 1991b; Jones et al., 2008) relative to medication-free treatment.

There has been particular interest in the relationship between methadone dose and neonatal outcomes for pregnant women in treatment for opioid dependence, as providers and patients share the same goals of wanting to effectively treat the mother while minimizing potential risks to the embryo and fetus. Higher maternal methadone dosing has been correlated with improved treatment retention and less concomitant illicit drug use (McCarthy et al., 2005; Seligman et al., 2010). Over the years, researchers have alternately found positive correlations between higher maternal methadone dose and more severe neonatal abstinence syndrome (NAS) [e.g., Dryden et al. (2009); Malpas et al. (1995); Wouldes et al. (2010)], questioned the relationship (O'Grady et al., in press), or found no correlations between the two variables [e.g., (Berghella et al. (2003); Pizarro et al. (2011); Seligman et al. (2010)]. These discrepant findings may be due to numerous methodological differences among the studies and subject populations. Indeed, one of the major methodological differences among the various studies is how 'higher' and 'lower' methadone dose has been defined. In many cases, such a definition is sample-specific, and based on a median split, mean split, or some form or low-medium-high division on methadone dose, typically at delivery or in the final trimester [see (Cleary et al., 2010) for various categorizations that have occurred in the published literature]. Such a course of action leads one study (Strauss et al., 1976) to define 'low' and 'high' dose groups as less than or equal to $20 \mathrm{mg}$ or more than $20 \mathrm{mg}$ methadone, respectively, while a second study (McCarthy et al., 2005) divides their sample into 'low' at less than $100 \mathrm{mg}$ and 'high' at $100 \mathrm{mg}$ or more of methadone. The interpretation of the impact of 'higher' doses of methadone then becomes problematic, and the comparison of the results across these studies then becomes virtually meaningless. Despite these significant limitations, a recent systematic literature review (Cleary et al., 2010) and meta-analysis of 29 studies that examined the relationship between maternal methadone dose and NAS from 1966-2009 concluded that when the analyses were limited either to prospective studies or to studies in which the measurement of NAS was conducted with a valid measure, the "severity of the NAS does not appear to differ according to whether mothers are on high- or low-dose methadone maintenance therapy" (p. 2071). 
Although less frequent, other research has examined the relationship between maternal methadone dose and neonatal outcomes other than NAS. Velez et al. (2009) found that maternal methadone dose was not correlated with scores on the NICU Network Neurobehavioral scale, a comprehensive measure of neurobehavioral functioning, in a sample of 77 infants. Gray et al. (2010) reported no relationship between maternal methadone dose at delivery and gestational age at birth, need for pharmacotherapy for NAS, peak NAS score, or birth parameters in a sample of 49 opioid-dependent pregnant women.

The Maternal Opioid Treatment: Human Experimental Research (MOTHER) study (Jones et al., 2010) examined maternal and neonatal clinical outcomes in a sample of 175 (131 of whom completed the trial and gave birth while maintained on study medication) opioiddependent pregnant women participating in a randomized clinical trial comparing methadone and buprenorphine. The MOTHER study is unique in several respects, notably because exclusionary criteria required participants to have no current abuse or dependence of either alcohol or benzodiazepines. Moreover, a contingency management approach in which patients earned monetary vouchers for providing urine samples negative for opioids and other illicit drugs was used in order to ensure that the participants maintained their drugfree status, which led to an extremely low level of use of concomitant drugs throughout the study that might have served to contaminate the relationship between maternal methadone dose levels and neonatal clinical outcomes.

The purpose of the present paper is twofold. The first purpose was to examine the relationship between methadone dose and neonatal clinical outcomes in the sample of 73 opioid-dependent pregnant women receiving methadone pharmacotherapy who participated in the MOTHER study. The second purpose was to examine differences in the neonatal outcomes between the neonates of these methadone-maintained participants who were $(n=41)$ and were not $(n=32)$ treated for NAS with morphine sulfate. Given the low level of use of concomitant drugs used by participants in MOTHER and the fact that the participants were provided with financial incentives to remain drug-free, data from the MOTHER study allow for an examination of these two issues, largely in the absence of poly-substance use on the part of the participants. Moreover, data from the MOTHER study has the advantage over most retrospective studies, as well as many prospective studies, in that the measurement of NAS was conducted by well-trained raters in a uniform manner and found to be highly reliable. Thus, the MOTHER study allows for an estimate of the relationship between methadone dose and neonatal clinical outcomes, unconfounded by other drugs of abuse, and for the estimation of differences between NAS treatment status (neonates untreated $v$. treated for NAS) on these same outcomes.

\section{Methods}

\subsection{The MOTHER Study}

The Maternal Opioid Treatment: Human Experimental Research (MOTHER) study (Jones et al., 2012; Jones et al., 2010) was a double-blind, double-dummy (i.e., both conditions received their respective medication and a placebo), flexible-dosing, parallel-group randomized clinical trial that provided comprehensive care to 175 opioid-dependent pregnant women who were given either methadone or buprenorphine. Results indicated that buprenorphine-exposed neonates required, on average, $89 \%$ less morphine to treat NAS, spent, on average, $43 \%$ less time in the hospital and, 58\% less time in the hospital being medicated for NAS than did the methadone-exposed neonates.

Complete details regarding the study can be found in Jones et al. (2012) and Jones et al. (2010). The following information provides an overview of the study germane to the present secondary analyses. 


\subsection{MOTHER Sites}

Neonatal outcome data were collected at 7 university hospital sites. However, sites were combined into three categories because the number of participants at some US sites was relatively small, and pooling the sites for the purposes of statistical analysis minimized the possibility that site heterogeneity due to small samples sizes might adversely impact the analyses: US Urban (Baltimore, MD; Philadelphia, PA: Detroit MI: Providence, RI) $v$. US Rural (Burlington, VT; Nashville, TN) v. European (Vienna).

\subsection{MOTHER Participants}

Opioid-dependent, singleton pregnant (6-30 weeks gestation) women meeting eligibility criteria were recruited. Participants were randomized to methadone or buprenorphine and received double-blind, double-dummy, study medication administered daily with sublingual tablets (buprenorphine or placebo) followed by oral liquid (methadone or placebo). A flexible dose range of 20 to $140 \mathrm{mg}$ of methadone was used in the methadone condition. To reduce concomitant drug use, patients earned monetary vouchers for providing urine samples thrice weekly that tested negative for other non-prescribed opioids and other illicit drugs. Of the 175 maternal participants, 131 delivered infants as part of the study, and of these 131, 73 were in the methadone condition with 41 neonates treated for NAS with morphine sulfate, and 32 infants receiving no pharmacotherapy for NAS. The secondary analyses reported in this paper use the data from the neonates of these 73 maternal participants.

\subsection{MOTHER Measures of Interest}

A modified Finnegan Scale (Finnegan et al., 1992) (MOTHER NAS Scale) was administered for a minimum of 10 days by trained staff to assess neonatal abstinence syndrome (NAS). The MOTHER NAS scale has a total of 28 items, with 19 items used for scoring and medication decisions. The maximum withdrawal score on the MOTHER NAS Scale is 42 . A NAS peak score was derived for each neonate, representing the highest NAS score the neonate obtained within the first 10 days after birth. The degree of agreement on NAS scale measures of the site raters and the expert rater who trained the site raters was assessed every 6 months and was evaluated by an intraclass correlation $[I C C(2,2)]$, which exceeded .94. The protocol specified that babies were to receive NAS observations for the first 10 postpartum days, regardless of NAS treatment. Hospital regulations did not allow some sites to keep non-treated babies in the hospital for more than 4 days. Thus, those babies received their multiple NAS observations on an outpatient basis. Neonates requiring pharmacotherapy for NAS were treated with morphine sulfate as inpatients for the duration of their therapy (Jones et al., 2012).

Information was also collected during the course of the trial on medication dose at delivery $(\mathrm{mg})$, and 8 additional measures of interest to the present study: total amount of morphine needed to treat NAS (mg), duration of neonatal hospital stay (days), duration of treatment for NAS (days), estimated gestational age at delivery (weeks), Apgar score at 5 minutes, and neonatal head circumference $(\mathrm{cm})$, length $(\mathrm{cm})$, and weight $(\mathrm{gm})$ at birth. [Apgar score at 1 minute was also collected and reported in Jones et al. (2010). Given the general conclusion that 1-minute Apgar scores are not predictive of neonatal future outcome (American Academy of Pediatrics Committee on Fetus and Newborn, 2006) an analysis of this variable is omitted from the current paper.]

\subsection{Statistical Analyses}

Because our assumption was that the following outcome variables were normally distributed, ordinary least squares regression analyses (Draper et al., 1998) were conducted 
for: Peak score on the MOTHER NAS scale during the assessment period, and infant head circumference, birth weight, and length. In contrast, our assumption was that the following outcomes followed a Poisson distribution in the population, so a Poisson regression model using Laplace approximation (Littell, 2006) was utilized for: Total amount of morphine needed to treat NAS (measured in mg), number of days medicated for NAS (days), duration of neonatal hospital stay (days), estimated gestational age at delivery (weeks), and Apgar scores at 5 minutes. The Poisson regression model included a random intercept term and an overdispersion parameter. In each Poisson regression analysis, the overdispersion parameter was tested for significance, and if it was not significant, the model was re-fitted omitting the dispersion parameter. (Laplace estimation was chosen because it generally shows better asymptotic behavior and less bias in small samples than does pseudo-likelihood estimation.)

Predictor variables in all models included a three-level fixed site factor. For those analyses that estimated the relationship between methadone dose at delivery and neonatal clinical outcomes, methadone dose at delivery was included as a continuous predictor as the primary effect of interest. For those analyses that examined the differences between NAS treatment status and neonatal clinical outcomes, a fixed effect representing NAS treatment status (untreated $v$. treated with morphine sulfate) was included in the statistical model as the primary effect of interest. NAS treatment status was necessarily only examined for 7 neonatal clinical outcomes, given that total amount of morphine needed to treat NAS and number of days medicated for NAS were, by definition, all zero in the group of neonates untreated for NAS. Unstandardized partial regression coefficients ( $b$ weights) and their standard errors are reported for the results for methadone dose, while model-derived estimated means are reported for NAS treatment status.

More complete details about MOTHER measures and statistical methods can be found in (Jones et al., 2010).

\section{Results}

Mean methadone dose at delivery was $84.9 \mathrm{mg}$ ( $S D=35.3$; range 20-140). Descriptive statistics for the 9 outcome measures can be found in Table 1 .

\subsection{Relationship between Methadone Dose and Neonatal Outcomes}

Results for the analyses of the 9 outcomes failed to reject the null hypothesis of no relationship between methadone dose and the respective outcome measure (all $p \mathrm{~s}>.05$ ). Parameter estimates and their standard errors for these analyses can be found in Table 2 .

However, all Poisson regression adjusted odds ratio $(A O R)$ estimates are quite close to 1 , suggesting the possibility that the statistical model was more complex than the data would allow for the 5 outcome variables assumed to follow a Poisson distribution. Because research has suggested that an overdispersed Poisson model can sometimes better be modeled as a negative binomial process, the analyses of these 5 variables were also conducted under the assumption that the outcome variable in question followed a negative binomial distribution in the population. Results indicated a failure to reject the null hypothesis (all $p s>.09$ ). As with the Poisson regression model, parameter estimates for the negative binomial model were again quite close to 1 , with narrow confidence intervals. In a final attempt to further simplify the statistical model, at the expense of the distributional assumption, the relationship between methadone dose at delivery and these 5 outcome measures was examined in an ordinary least squares regression model, with the same result (all $p s>.17)$. 
Finally, only 41/73 neonates were treated for NAS, and so 32 neonates necessarily had scores of 0 for total amount of morphine needed to treat NAS and number of days treated for NAS (hence the need for an overdispersed Poisson model). Both Poisson and negative binomial regression analyses for these two outcome variables that included observations only from these 41 neonates treated for NAS found no relationship between methadone dose at delivery and either outcome (all $p \mathrm{~s}>.4$ ).

\subsection{Relationship between NAS Treatment Status and Neonatal Outcomes}

For those infants treated for NAS, the mean total amount of morphine for NAS (mg) was 33.7 ( $S D=64.8$ ), with the mean dose of morphine per $\mathrm{kg}$ of body weight at the initiation of treatment for NAS equal to $0.028(S D=0.022)$ and the mean highest dose of morphine per $\mathrm{kg}$ of body weight equal to 0.032 ( $S D=0.030$ ). The mean duration of treatment for NAS (days) was 21.7 ( $S D=17.9)$. (One case is missing data for these outcomes due to neonatal demise.)

Table 3 presents descriptive statistics for the 7 remaining outcomes in the untreated ( $n=32$ ) and treated $(n=41)$ for NAS groups. Significant mean differences were found between these two groups for duration of neonatal hospital stay, $F(1,69)=19.7, p<.001,[M=9.8$ days $(S E=2.1)$ v. $M=23.5$ days $(S E=2.4)$, respectively], NAS peak score, $F(1,68)=71.4, p<$. 001, [ $M=8.1(S E=0.7)$ v. $M=16.1(S E=0.6)$, respectively], and neonatal weight at birth, $F(1,69)=4.7, p<.04,[M=2735.8 \mathrm{gm}(S E=96.6)$ v. $M=3015.9 \mathrm{gm}(S E=87.3)$, respectively]. All other tests of mean differences between the NAS treatment status groups were nonsignificant (all $p \mathrm{~s}>.07$ ).

\section{Discussion}

Findings of the present study, in need of confirmation by future prospective studies, extend the reach of the recent systematic review (Cleary et al., 2010) beyond the scope of NAS, and replicate and extend the findings of Gray et al. (2010). The failure to find any support for a relationship between maternal methadone dose at delivery and the 9 neonatal clinical outcomes was examined in the context in which the Type I error rate was set at .05, maximizing our chances of rejecting $\mathrm{H}_{0}$ and therefore finding support for a relationship between maternal methadone dose at delivery and any one or more of the neonatal clinical outcomes.

Furthermore, the relationship between methadone dose and 5 of the neonatal outcomes were tested under a variety of distributional assumptions for these variables. Despite such a liberal approach to hypothesis testing, no support was found for a relationship between methadone dose and neonatal outcomes for any 1 of the 9 variables. Moreover, it should be noted that this failure to reject any 1 of the 9 null hypotheses occurred in a sample of women who were not abusing, in any significant way, other substances that could serve to either mask or boost a relationship between the variables in question. In other words, testing 9 interdependent hypotheses in a way that favored rejection of one or more null hypotheses failed to yield a significant finding. Taken together, these findings suggest that there is likely no relationship between maternal methadone dose at delivery and any of several clinically important neonatal outcomes, including NAS severity.

It is reasonable at this point to ask why there are findings in the literature contrary to this conclusion, as noted in the introduction. There is, of course, the obvious 'first' conclusion that the studies in question represent Type I error. Certainly this is a possibility for some studies, but such a conclusion is both too quick and likely too simplistic. It is probable there are several factors operating that have contributed (and perhaps will continue to contribute) to this confusion. First, it is quite possible that there are differences in the populations from which the samples have been drawn. There may be geographical differences in the maternal 
and infant subjects and drug use patterns, and in local treatment clinic practices that might lead to differences in the population of opioid-dependent pregnant women seeking treatment, and/or the extent and nature of treatment provided to them. Optimal treatment for opioid-dependent pregnant women offers methadone pharmacotherapy in the context of comprehensive care including obstetric care and drug treatment; however, such care is not always available, particularly in rural areas. The combination of different populations seeking treatment for different reasons and being provided different treatments could certainly operate to either ameliorate or amplify the relationship between maternal methadone dose at delivery and neonatal outcomes.

Second, there is the obvious issue of how maternal methadone dose at delivery has been defined by the various researchers in this area that has likely impeded the ability to determine whether there is any relationship between maternal methadone dose at delivery and outcome. Researchers have 'crudely classified observations', dividing their samples into low and high groups by methods such as the median split, as noted in the Introduction. Such classification schemes are ad hoc, and greatly impede the ability of reviewers and researchers to compare results across studies. Moreover, as Cohen (1983) has convincingly shown: "Assuming bivariate normality with correlation $r$, dichotomizing one variable at the mean results in the reduction in variance accounted for to $.647 r^{2} \ldots$ ". resulting in "reduction in statistical power equivalent to discarding $38 \% \ldots$ of the cases under representative conditions" (p. 249). Barring some compelling argument for an exception to this conclusion, the only reasonable approach would be to treat maternal methadone dose at delivery as a continuous variable, and correlate it with the outcome variable(s). Such an approach has the added virtue of answering the question of whether or not there is a dose-response relationship between methadone and the outcome(s) of interest, and estimating the rate of change associated with this relationship.

Third, there is neither standardized assessment for NAS measurement nor its treatment. NAS measurement is highly variable, both between tools and in use of the same tool, and depends on subjective assessments by a variety of assessors with variable levels of skill and/ or experience with substance exposed infants. The potential interchangeability among different tools has not been examined. NAS treatment is also widely variable, with variable non-pharmacologic measures applied or not applied, different medications used, and different dosing strategies employed. All of these factors likely lead to differences in statistical conclusion validity among the studies that have utilized them.

Fourth, there is no evidence that maternal dose of methadone correlates with other biological samples germane to this discussion, which may help to explain the fact that maternal methadone dose at delivery is not the strong predictor of neonatal clinic outcomes as might first have been thought. Maternal dose did not correlate with maternal serum concentrations of methadone in a sample of recently postpartum women (Jansson et al., 2007). The lack of a significant relationship between maternal methadone dose and meconium concentrations of methadone and its metabolites and NAS severity (Gray et al., 2010; Kuschel et al., 2004) suggests that neonates differ in the rate of elimination of methadone from their systems. Metabolism of methadone by the placenta (Nanovskaya et al., 2004), fetus (Kuschel et al., 2004), and infant (Rosen et al., 1976) are likely to play a role in the variable expression of NAS among opioid-exposed infants, and may additionally be affected by other factors such as genetics (Oei et al., 2012), although the nature of these relationships have yet to be fully elucidated.

Finally, it is imperative to note that methadone, like any medication given during the perinatal period, has risks and benefits. Results of the present study do not lend support to the contention that limiting the dose of methadone to treat pregnant women will reduce the 
possibility of poor neonatal outcomes. Indeed, such a position contradicts national clinical guidelines [e.g., Center for Substance Abuse Treatment. (2005); New South Wales Department of Health, (2006)] and may create unintended yet harmful radiating effects for the mother, the fetus, and the neonate.

The findings that the group treated for NAS had a higher mean NAS peak score and spent a longer time in the hospital on average than did the group not treated for NAS are hardly surprising. Both of these outcomes are likely the result, in large part, of the need for NAS treatment. The finding that the untreated group weighed less at birth than the treated group is likewise unsurprising, given it had been reported in a study predicting NAS treatment in the entire MOTHER sample (Kaltenbach et al., 2012). It should be noted that the ratio of females:males and preterm:term for the two groups was roughly equivalent, so this result is not due to differences in gender or prematurity in the two groups. Certainly this finding merits attention in future research.

\subsection{Limitations}

There is the clear concern that the present study is based on a relatively small sample of women who are atypical of the majority of opioid-dependent pregnant women in methadone pharmacotherapy. For example, Dryden and colleagues (Dryden et al., 2009) found a positive relationship between methadone dose and NAS incidence in a retrospective study of 444 methadone-maintained women. However, many of the Dryden et al. participants were poly-substance-using, and $93 \%$ of the neonates receiving pharmacotherapy for NAS had been exposed to prenatal poly-substance abuse. In contrast, the MOTHER sample showed no current abuse or dependence of either alcohol or benzodiazepines and generally used cocaine and other drugs at low levels, if at all. Moreover, participants were offered financial incentives to remain drug-free throughout their time in the study. Thus, the extent to which findings from the present study generalize to the larger population of opioid-dependent pregnant women in methadone pharmacotherapy is unknown, and requires further investigation. However, it is also the case that the present sample allows for the examination of the relationship between methadone dose and neonatal outcomes without the potentially confounding factor of poly-substance use.

\subsection{Conclusions}

Opioid dependence rarely occurs in the absence of confounding bio-psycho-social issues. Thus, the effects of any drug or medication - including methadone - on mothers and infants are best understood in the context of complex life challenges that most opioid-dependent pregnant patients face and other exposures (i.e. other licit or illicit medications/drugs, nicotine, alcohol, maternal stress, etc.) that the fetus experiences. The field would be best served by future research focused on understanding and ameliorating the underlying factors which lead women to initiate and maintain drug use, and the roles such factors might play in both maternal and neonatal response to medications and their longer-term outcomes. Understanding such factors could allow more focused and specific treatment for the highly complex and vulnerable population of opioid-dependent women and their children.

\section{Acknowledgments}

Role of Funding Source

Author LMJ was supported by grant RO1 DA031689 from the National Institute on Drug Abuse (NIDA) during the preparation of this paper. NIDA played no role in the: 1) study design; 2) collection, analysis, and interpretation of data; 3 ) writing of the report; and 4) decision to submit or where to submit the paper for publication. 


\section{References}

American Academy of Pediatrics Committee on Fetus and Newborn. The Apgar score. Pediatrics. 2006; 117:1444-7. [PubMed: 16585348]

Berghella V, Lim PJ, Hill MK, Cherpes J, Chennat J, Kaltenbach K. Maternal methadone dose and neonatal withdrawal. Am J Obstet Gynecol. 2003; 189:312-7. [PubMed: 14520184]

Center for Substance Abuse Treatment. Treatment Improvement Protocol \#43. Substance Abuse and Mental Health Services Administration; Rockville, MD: 2005. Medication-assisted treatment for opoid addiction in opioid treatment programs.

Cleary BJ, Donnelly J, Strawbridge J, Gallagher PJ, Fahey T, Clarke M, Murphy DJ. Methadone dose and neonatal abstinence syndrome-systematic review and meta-analysis. Addiction. 2010; 105:2071-84. [PubMed: 20840198]

Cohen J. The cost of dichotomization. Appl Psyc Measure. 1983; 7:249-53.

Connaughton JF, Reeser D, Schut J, Finnegan LP. Perinatal addiction: outcome and management. Am J Obstet Gynecol. 1977; 129:679-86. [PubMed: 303464]

National Consensus Development Panel on Effective Medical Treatment of Opiate Addiction. Effective Medical Treatment of Opiate Addiction. JAMA. 1998; 280:1936-43. [PubMed: 9851480]

Draper, NR.; Smith, H. Applied Regression Analysis. 3rd ed. Wiley; New York: 1998.

Dryden C, Young D, Hepburn M, Mactier H. Maternal methadone use in pregnancy: factors associated with the development of neonatal abstinence syndrome and implications for healthcare resources. BJOG. 2009; 116:665-71. [PubMed: 19220239]

Finnegan LP. Perinatal substance abuse: comments and perspectives. Semin Perinatol. 1991a; 15:3319. [PubMed: 1948145]

Finnegan LP. Treatment issues for opioid-dependent women during the perinatal period. J Psychoactive Drugs. 1991b; 23:191-201. [PubMed: 1765892]

Finnegan, LP.; Kaltenbach, K. Neonatal abstinence syndrome. In: Hoekelman, RA.; Friedman, SB.; Nelson, NM., et al., editors. Primary Pediatric Care. Mosby; St. Louis: 1992. p. 1367-78.

Gray TR, Choo RE, Concheiro M, Williams E, Elko A, Jansson LM, et al. Prenatal methadone exposure, meconium biomarker concentrations and neonatal abstinence syndrome. Addiction. 2010; 105:2151-9. [PubMed: 20854338]

Jansson LM, Choo RE, Harrow C, Velez M, Schroeder JR, Lowe R, Huestis MA. Concentrations of methadone in breast milk and plasma in the immediate perinatal period. J Hum Lact. 2007; 23:184-90. [PubMed: 17478871]

Jones HE, Fischer G, Heil SH, Kaltenbach K, Martin PR, Coyle MG, et al. Maternal Opioid Treatment: Human Experimental Research (MOTHER): Approach, Issues, and Lessons Learned. Addiction. 2012; 107(Suppl 1):28-35. [PubMed: 23106924]

Jones HE, Haug N, Silverman K, Stitzer M, Svikis D. The effectiveness of incentives in enhancing treatment attendance and drug abstinence in methadone-maintained pregnant women. Drug Alcohol Depend. 2001; 61:297-306. [PubMed: 11164694]

Jones HE, Kaltenbach K, Heil SH, Stine SM, Coyle MG, Arria AM, et al. Neonatal abstinence syndrome after methadone or buprenorphine exposure. N Engl J Med. 2010; 363:2320-31. [PubMed: 21142534]

Jones HE, O’Grady KE, Malfi D, Tuten M. Methadone maintenance vs. methadone taper during pregnancy: maternal and neonatal outcomes. Am J Addict. 2008; 17:372-86. [PubMed: 18770079]

Jones, HE.; Tuten, M.; Keyser-Marcus, L.; Svikis, D. Specialty treatment for women. In: Strain, EC.; Stitzer, ML., editors. Methadone treatment for opioid dependence. Johns Hopkins University Press; Baltimore: 2006. p. 455-484.

Kaltenbach K, Berghella V, Finnegan L. Opioid dependence during pregnancy. Effects and management. Obstet Gynecol Clin North Am. 1998; 25:139-151. [PubMed: 9547764]

Kaltenbach K, Holbrook AM, Coyle MG, Heil SH, Salisbury AL, Stine SM, et al. Predicting treatment for neonatal abstinence syndrome in infants born to women maintained on opioid agonist medication. Addiction. 2012; 107(Suppl 1):45-52. [PubMed: 23106926]

Kandall SR, Albin S, Gartner LM, Lee KS, Eidelman A, Lowinson J. The narcotic-dependent mother: fetal and neonatal consequences. Early Hum Dev. 1977; 1:159-69. [PubMed: 617308] 
Kuschel CA, Austerberry L, Cornwell M, Couch R, Rowley RS. Can methadone concentrations predict the severity of withdrawal in infants at risk of neonatal abstinence syndrome? Arch Dis Child Fetal Neonatal Ed. 2004; 89:F390-3. [PubMed: 15321955]

Laken MP, Ager JW. Effects of case management on retention in prenatal substance abuse treatment. Am J Drug Alcohol Abuse. 1996; 22:439-48. [PubMed: 8841690]

Littell, RC. SAS for mixed models. 2nd ed. SAS Institute; Cary, N.C.: 2006.

Malpas TJ, Darlow BA, Lennox R, Horwood LJ. Maternal methadone dosage and neonatal withdrawal. Aust N Z J Obstet Gynaecol. 1995; 35:175-7. [PubMed: 7677682]

McCarthy JJ, Leamon MH, Parr MS, Anania B. High-dose methadone maintenance in pregnancy: maternal and neonatal outcomes. Am J Obstet Gynecol. 2005; 193:606-10. [PubMed: 16150249]

Nanovskaya TN, Deshmukh SV, Nekhayeva IA, Zharikova OL, Hankins GD, Ahmed MS. Methadone metabolism by human placenta. Biochem Pharmacol. 2004; 68:583-91. [PubMed: 15242824]

New South Wales Department of Health. National Clinical Guidelines for the Management of Drug Use During Pregnancy, Birth and the Early Development Years of the Newborn. New South Wales Department of Health; Sydney: 2006.

Oei JL, Xu HX, Abdel-Latif ME, Vunnam K, Al-Amry A, Clews S, et al. Dopamine D2 receptor gene polymorphisms in newborn infants of drug-using women. Arch Dis Child Fetal Neonatal Ed. 2012; 97:F193-8. [PubMed: 21948329]

O'Grady KE, Jones HE, Jansson LM, Kaltenbach K. Maternal methadone dose during pregnancy and infant clinical outcome: Further considerations. Neurotoxicol Teratol. in press.

Pizarro D, Habli M, Grier M, Bombrys A, Sibai B, Livingston J. Higher maternal doses of methadone does not increase neonatal abstinence syndrome. J Subst Abuse Treat. 2011; 40:295-8. [PubMed: 21255960]

Rosen TS, Pippenger CE. Pharmacologic observations on the neonatal withdrawal syndrome. J Pediatr. 1976; 88:1044-8. [PubMed: 1271174]

Schmid M, Kuessel L, Klein K, Metz V, Fischer G, Krampl-Bettelheim E. First-trimester fetal heart rate in mothers with opioid addiction. Addiction. 2010; 105:1265-8. [PubMed: 20642509]

Seligman NS, Almario CV, Hayes EJ, Dysart KC, Berghella V, Baxter JK. Relationship between maternal methadone dose at delivery and neonatal abstinence syndrome. J Pediatr. 2010; 157:42833. [PubMed: 20472252]

Strauss ME, Andresko M, Stryker JC, Wardell JN. Relationship of neonatal withdrawal to maternal methadone dose. Am J Drug Alcohol Abuse. 1976; 3:339-45. [PubMed: 1032747]

Svikis DS, Lee JH, Haug NA, Stitzer ML. Attendance incentives for outpatient treatment: effects in methadone- and nonmethadone-maintained pregnant drug dependent women. Drug Alcohol Depend. 1997; 48:33-41. [PubMed: 9330919]

Velez ML, Jansson LM, Schroeder J, Williams E. Prenatal methadone exposure and neonatal neurobehavioral functioning. Pediatr Res. 2009; 66:704-9. [PubMed: 19690513]

Wouldes TA, Woodward LJ. Maternal methadone dose during pregnancy and infant clinical outcome. Neurotoxicol Teratol. 2010; 32:406-13. [PubMed: 20102736] 


\section{Highlights}

- We conducted secondary analyses of MOTHER data (H. E. Jones et al., New England Journal of Medicine, 2010).

- These analyses found no relationship between maternal methadone dose and 9 neonatal outcomes.

- The conclusion of a methadone dose-response relationship with neonatal outcomes is unwarranted. 


\section{Table 1}

Descriptive Statistics for the 9 Neonatal Clinical Outcomes from the MOTHER Methadone Condition participants $(N=73)$

\begin{tabular}{|c|c|c|c|}
\hline Variable & $M$ & $S D$ & Range \\
\hline Total amount of morphine for NAS ${ }^{\dagger}(\mathrm{mg})$ & 19.2 & 51.5 & $0-354.5$ \\
\hline Duration of treatment for NAS ${ }^{\dagger}$ (days) & 12.3 & 17.2 & $0-76$ \\
\hline Duration of neonatal hospital stay (days) & 18.3 & 17.2 & $2-79$ \\
\hline Gestational age at delivery (weeks) & 38.0 & 2.3 & $32-41$ \\
\hline Apgar score at 5 minutes & 8.9 & 1.0 & $5-10$ \\
\hline Peak NAS score ${ }^{\dagger}$ & 12.8 & 5.5 & $3-26$ \\
\hline Neonatal weight at birth (gm) & 2880.0 & 546.1 & $1440-3742$ \\
\hline Neonatal length at birth $(\mathrm{cm})^{\dagger}$ & 47.8 & 4.6 & $20-54$ \\
\hline Neonatal head circumference at birth $(\mathrm{cm})^{\dagger}$ & 33.0 & 2.1 & $24-36$ \\
\hline
\end{tabular}

Notes. NAS = Neonatal Abstinence Syndrome. Means do not equal values found in Table 2 of Jones et al. (2010), because the latter means were model-derived marginal means from a model that includes the buprenorphine condition, and were adjusted for site.

One case is missing data for this variable due to neonatal demise. 
Table 2

Estimates from the Regression Analyses Examining the Relationship between Methadone Dose and 9 Neonatal Clinical Outcomes from the MOTHER Methadone Condition participants $(N=73)$

\begin{tabular}{|c|c|c|c|c|c|}
\hline Outcome Variable & $b$ & $S E$ & $A O R$ & $95 \% C I$ & $P$ \\
\hline $\begin{array}{l}\text { Total amount of morphine for } \\
\text { NAS }^{\dagger}(\mathrm{mg})\end{array}$ & .01 & .01 & 1.01 & $1.00,1.03$ & .09 \\
\hline $\begin{array}{l}\text { Duration of treatment for NAS } \\
\text { (days) }\end{array}$ & .01 & .01 & 1.01 & $1.00,1.03$ & .051 \\
\hline $\begin{array}{l}\text { Duration of neonatal hospital } \\
\text { stay (days) }\end{array}$ & .00 & .00 & 1.00 & $1.00,1.01$ & .14 \\
\hline $\begin{array}{l}\text { Gestational age at delivery } \\
\text { (weeks) }\end{array}$ & .00 & .00 & 1.00 & $1.00,1.00$ & .84 \\
\hline Apgar score at 5 minutes & .00 & .00 & 1.00 & $1.00,1.00$ & .86 \\
\hline NAS peak score ${ }^{t}$ & .04 & .02 & & & .07 \\
\hline $\begin{array}{l}\text { Neonatal head circumference at } \\
\text { birth }(\mathrm{cm})^{\dagger}\end{array}$ & .00 & .01 & & & .80 \\
\hline Neonatal weight at birth (gm) & 1.55 & 2.12 & & & .47 \\
\hline Neonatal length at birth $(\mathrm{cm})^{\dagger}$ & .01 & .01 & & & .57 \\
\hline
\end{tabular}

Notes. Predictor variables included maternal methadone dose at delivery $(\mathrm{mg})$ and a three-level fixed site factor. NAS $=$ Neonatal Abstinence Syndrome. $S E=$ Standard Error. $A O R=$ Adjusted Odds Ratio (adjusted for the fixed site factor). $95 \% C I=95 \%$ Confidence Interval. Estimates for Total amount of morphine for NAS and Duration of treatment for NAS were derived from an overdispersed Poisson regression model, while estimates for Duration of neonatal stay, Gestational age at delivery, and Apgar scores at 5 minutes were derived for a Poisson regression model that omitted the overdispersion term, because the test of the need for an overdispersion parameter was nonsignificant for these 3 outcomes. Estimates for NAS peak score and neonatal head circumference, weight, and length at birth were derived from ordinary least squares regression.

${ }^{\dagger}$ One case is missing data for this variable due to neonatal demise. 
Table 3

Model-derived Estimated Means and Standard Errors for the 7 Neonatal Clinical Outcomes from the MOTHER Methadone Condition participants Treated $(n=41)$ and Untreated $(n=32)$ for Neonatal Abstinence Syndrome

\begin{tabular}{|c|c|c|c|c|c|}
\hline \multirow[b]{2}{*}{ Outcome Variable } & \multicolumn{2}{|c|}{$\begin{array}{l}\text { Treated for NAS } \\
(n=41)\end{array}$} & \multicolumn{2}{|c|}{$\begin{array}{l}\text { Untreated for NAS } \\
(n=32)\end{array}$} & \multirow[b]{2}{*}{$\boldsymbol{P}$} \\
\hline & $\boldsymbol{M}$ & $S E$ & $M$ & $S E$ & \\
\hline Duration of neonatal hospital stay (days) & 23.5 & 2.4 & 9.8 & 2.1 & $<.001$ \\
\hline Gestational age at delivery (weeks) & 38.2 & 1.0 & 37.8 & 1.1 & .75 \\
\hline Apgar score at 5 minutes & 9.0 & 0.5 & 8.9 & 0.5 & .98 \\
\hline Peak NAS score ${ }^{\dagger}$ & 16.1 & 0.6 & 8.1 & 0.7 & $<.001$ \\
\hline Neonatal weight at birth (gm) & 3015.9 & 87.3 & 2735.8 & 96.6 & .04 \\
\hline Neonatal length at birth $(\mathrm{cm})^{\dagger}$ & 48.8 & 0.7 & 46.7 & 0.8 & .07 \\
\hline Neonatal head circumference at birth $(\mathrm{cm})^{\dagger}$ & 33.4 & 0.3 & 32.6 & 0.4 & .15 \\
\hline
\end{tabular}

Notes. Predictor variables in the model included a fixed effect represented NAS treatment status and a three-level fixed site factor. NAS $=$ Neonatal Abstinence Syndrome. $M=$ Model-derived estimated mean. (Means are adjusted for the fixed site factor.) $S E=$ Standard Error.Means for Duration of neonatal stay, Gestational age at delivery, and Apgar scores at 5 minutes were derived for a Poisson regression model that omitted an overdispersion term, because the test of the need for an overdispersion parameter was nonsignificant for these 3 outcomes. The estimates for NAS peak score and neonatal head circumference, weight, and length at birth were derived from ordinary least squares regression.

One case is missing data for this variable due to neonatal demise. 